\title{
Computing the Distribution Function of the Number of Renewals
}

\author{
Mohan L. Chaudhry, Xiaofeng Yang, Boon Ong \\ Department of Mathematics and Computer Science, Royal Military College of Canada, Kingston, Canada \\ Email: chaudhry-ml@rmc.ca, xiaofeng.yang@rmc.ca, ong-b@rmc.ca
}

Received April 20, 2013; revised May 25, 2013; accepted June 15, 2013

Copyright (C) 2013 Mohan L. Chaudhry et al. This is an open access article distributed under the Creative Commons Attribution License, which permits unrestricted use, distribution, and reproduction in any medium, provided the original work is properly cited.

\begin{abstract}
The method of Laplace transforms is used to find the distribution function, mean, and variance of the number of renewals of a renewal process whose inter-arrival time distribution has a rational Laplace transform. Where the Laplace transform is not rational, we use the Padé approximation method. We apply our method to certain examples and the results are compared to those reported by other researchers.
\end{abstract}

Keywords: Number of Renewals; Rational Approximation; Padé

\section{Introduction}

Renewal and reliability theories are powerful modeling tools in many applications, considering, for example, a series of renewals during a time interval $(0, t]$ with the inter-renewal times having certain distributions. The interest, usually, is to find the distribution or the moments of the number of renewals during the interval $(0, t]$. Although the theoretical aspects of renewal theory have been discussed in various books such as Cox [1] and Feller [2,3], not much seem to have been done in applying the theoretical results to practice for lack of availability of easily computable results. Using the cubic splining algorithm to compute the recursively-defined convolution integrals that appear in renewal theory, Baxter et al. [4] is able to construct some tables for the mean and variance of the number of renewals for different inter-renewal time distributions with varying parameters. A straightforward method to compute the convolution integral is to use the Laplace transform (LT) method. Using the root-finding method, Chaudhry [5] develops a unified method to compute the mean and variance of the number of renewals. Though the means and variances are useful for many applications, this paper goes a step further and deals with computing the distribution of the number of renewals from which one can get more information than from the mean and variance.

There are several methods that can be used for the numerical inversion of generating functions (GFs); see the excellent reviews on the numerical inversion (of GFs as well as of LTs) by Abate et al. [6-9]. Some researchers have been critical of using the method of roots, see, e.g. Cox and Smith [10] and Kleinrock [11]. In view of this, this paper serves another useful purpose and shows that with the availability of high precision in current software packages, the roots can be found successfully and used to invert the transforms. Further, using the roots method, the results can be first given in an analytically explicit form and then used to find the final results.

\section{Theory}

\subsection{Problem Description and Method of Laplace Transforms}

Let $X_{1}, X_{2}, \cdots$ be a governing sequence of independently identically distributed (i.i.d.) inter-renewal times for the renewal process $\{N(t), t \geq 0\}$, where $N(t)$ denotes the number of renewals in $(0, t]$. Let $X$ represent the generic inter-renewal time with cumulative distribution function (CDF)

$$
F(t)= \begin{cases}P(X \leq t), & t \geq 0 \\ 0, & t<0\end{cases}
$$

and probability density function (pdf) $f(t)$.

Define the probability mass function (pmf)

$P_{n}(t)=P(N(t)=n), n=0,1,2, \cdots$. Let

$S_{n}=\sum_{i=1}^{n} X_{i}, n \geq 1, S_{0}=0$. For $n \geq 0, S_{n}$ represents the time when the $n$-th renewal occurs. Define $F_{n}(t)=P\left(S_{n} \leq t\right)$, with $F_{0}(t)=1$. Then 


$$
F_{n}(t)=F^{* n}(t), n=0,1,2, \cdots
$$

is the $n$-fold convolution of $F(t)$ with itself, and (see Chaudhry and Templeton [12])

$$
P_{n}(t)=F_{n}(t)-F_{n+1}(t), t \geq 0, n \geq 0
$$

Let $\tilde{f}(s)$ and $\tilde{p}_{n}(s)$ be the Laplace-Stieltjes transform (LST) of $F(t)$ and $P_{n}(t)$, respectively, defined by $\tilde{f}(s)=\int_{0}^{+\infty} \mathrm{e}^{-s t} \mathrm{~d} F(t)$ and $\tilde{p}_{n}(s)=\int_{0}^{+\infty} \mathrm{e}^{-s t} \mathrm{~d} P_{n}(t)$. Taking the LST on both sides of Equation (1), we get

$$
\tilde{p}_{n}(s)=\tilde{f}^{n}(s)-\tilde{f}^{n+1}(s)=\tilde{f}^{n}(s)(1-\tilde{f}(s))
$$

and hence

$$
P_{n}(t)=\mathcal{L}^{-1}\left[\frac{\tilde{p}_{n}(s)}{s}\right]=\mathcal{L}^{-1}\left[\frac{\tilde{f}^{n}(s)(1-\tilde{f}(s))}{s}\right] .
$$

\subsection{Inversion of Laplace Transforms}

\subsection{1. $\tilde{f}(s)$ Is a Rational Function}

The inverse LT Equation (3) can be obtained analytically using partial fractions. Let

$$
\tilde{f}(s)=\frac{N(s)}{D(s)}
$$

where $D(s)$ and $N(s)$ are polynomials of degree $k$ and at most $k$, respectively. Then by Equation (2), we have

$$
\frac{\tilde{p}_{n}(s)}{s}=\frac{N^{n}(s)[D(s)-N(s)]}{s D^{n+1}(s)}
$$

which is a rational function.

Without loss of generality, we assume that the equation $D(s)=0$ has $k$ distinct roots $s_{1}, s_{2}, \cdots, s_{k}$. Since $\tilde{f}(0)=1, N(s)$ and $D(s)$ have the same constant terms, $D^{n+1}(s)=\left(s-s_{1}\right)^{n+1}\left(s-s_{2}\right)^{n+1} \cdots\left(s-s_{k}\right)^{n+1}$. And Equation (4) can be expressed in partial fractions as

$$
\frac{\tilde{p}_{n}(s)}{s}=\sum_{j=1}^{k} \sum_{i=1}^{n+1} \frac{A_{j, i}}{\left(s-s_{j}\right)^{i}}
$$

where the constant coefficient $A_{j, i}$ is given by

$$
A_{j, i}=\frac{1}{(i-1) !} \frac{\mathrm{d}^{(i-1)}}{\mathrm{d} s^{i-1}}\left[\frac{\tilde{p}_{n}(s)}{s}\left(s-s_{j}\right)^{i}\right]
$$

The final inversion can be written as

$$
P_{n}(t)=\sum_{j=1}^{k} \sum_{i=1}^{n+1} \frac{A_{j, i}}{(i-1) !} t^{i-1} \mathrm{e}^{s_{j} t}
$$

The case when $D(s)=0$ has repeated roots can be dealt with similarly.

\subsection{2. $\tilde{f}(s)$ Is Not a Rational Function}

We use the Padé approximation method. Assume

$$
\tilde{f}(s)=\sum_{n=0}^{+\infty}(-1)^{n} \frac{1}{n !} M_{n} s^{n}
$$

where

$$
M_{n}=\int_{0}^{+\infty} x^{n} \mathrm{~d} F(x)
$$

is the $n$-th moment of the inter-renewal time. We can find a rational approximation function

$$
\hat{f}(s)=\frac{N(s)}{D(s)}=\frac{\sum_{n=0}^{K} b_{n} s^{n}}{\sum_{n=0}^{L} a_{n} s^{n}}
$$

where $N(s)$ and $D(s)$ are polynomials of degree $K$ and $L$, respectively with undetermined coefficients $b_{n}$ and $a_{n}$, such that the first $K+L$ moments of $\tilde{f}(s)$ are equal to those of $\tilde{f}(s)$. We denote the above Padé approximation as $[K / L]$ (Baker et al. [13]). In practice, $a_{0}$ is set to one and $K$ and $L$ are chosen by trial and error. Equating the moments and formulating the simultaneous equations, the coefficients $b_{n}$ and $a_{n}$ are uniquely determined (Baker et al. [13], Harris [14]).

Use of continued fractions is another way to obtain an approximate rational function $\tilde{f}(s)$. The method is, in fact, a special case of the Padé method (Baker et al. [13]).

There are times when using Padé method directly is not possible or does not give the desired results. For example, the Pareto distribution has an infinite second moment, and directly equating the moments cannot be done. For the lognormal distribution, the waveform of the Padé approximated distribution function shows quite large errors. The solution is to have a two-step approximation. The first step is to use line segments to approximate the distribution function. In the second step, the Padé method is used to generate a rational LT. By adjusting the parameters in these two steps, $\tilde{f}(s)$ can be obtained with the desired properties. We will illustrate this technique in the examples.

\subsection{Verification of the Distribution}

The expected number of renewals of $N(t)$ in $(0,1]$ is given by

$$
E[N(t)]=\sum_{n=0}^{+\infty} n P_{n}(t)
$$

and its variance by

$$
V[N(t)]=\sum_{n=0}^{+\infty} n^{2} P_{n}(t)-E[N(t)]^{2}
$$

In all our examples, the $P_{n}(t)$ obtained in Equation (5) is used to compute $E[N(t)]$ and $V[N(t)]$, and 
are checked against the results obtained by (Chaudhry [5]) using

$$
E[N(t)]=\mathcal{L}^{-1}\left[\frac{\tilde{f}(s)}{s(1-\tilde{f}(s))}\right]
$$

and

$$
V[N(t)]=\mathcal{L}^{-1}\left[\frac{\tilde{f}(s)(1+\tilde{f}(s))}{s(1-\tilde{f}(s))^{2}}\right] .
$$

In addition, we also match the resulting mean and variance with those of Baxter et al. [4].

\section{Examples}

\subsection{Erlang Distribution}

We first consider the simple case where $f(t)$ follows the Erlang distribution with the shape parameter equal to 2 , and we can get exact analytical results.

The pdf and CDF of $f(t)$ are given, respectively, by

$$
f(t)=\lambda^{2} t \mathrm{e}^{-\lambda t}
$$

and

$$
F(t)=1-\mathrm{e}^{-\lambda t}(1+\lambda t)
$$

Thus,

$$
\tilde{f}(s)=\frac{\lambda^{2}}{(s+\lambda)^{2}} .
$$

We get

$$
\begin{aligned}
& \tilde{p}_{n}(s)=\frac{\lambda^{2 n} s^{2}}{(s+\lambda)^{2 n+2}}+\frac{2 \lambda^{2 n+1} s}{(s+\lambda)^{2 n+2}} \\
& P_{n}(t)=\frac{\mathrm{e}^{-\lambda t}(\lambda t)^{2 n}}{(2 n+1) !}(\lambda t+(2 n+1)),
\end{aligned}
$$

$$
E[N(t)]=\mathcal{L}^{-1}\left[\frac{\lambda^{2}}{s^{2}(s+2 \lambda)}\right]=-\frac{1}{4}+\frac{1}{2} \lambda t+\frac{1}{4} \mathrm{e}^{-2 \lambda t},
$$

and

$$
\begin{aligned}
V[N(t)] & =\mathcal{L}^{-1}\left[\frac{\lambda^{2}\left(s^{2}+2 \lambda s+2 \lambda^{2}\right)}{s^{2}(s+2 \lambda)^{2}}\right] \\
& =\frac{1}{16}+\frac{1}{4} \lambda t-\frac{1}{2} \mathrm{e}^{-2 \lambda t} \lambda t-\frac{1}{16} \mathrm{e}^{-4 \lambda t} .
\end{aligned}
$$

It may be remarked that the analytic expression for the mean has been incorrectly reported by Parzen $[15, \mathrm{p}$. 177].

\subsection{Mixed Generalized Erlang Distribution}

The pdf and LST of a Mixed Generalized Erlang (MGE) distribution are given, respectively, by

$$
f(t)=\sum_{j=1}^{k} c_{j} \frac{\lambda^{j} t^{j-1} \mathrm{e}^{-\lambda t}}{(j-1) !}, t \geq 0
$$

and

$$
\tilde{f}(s)=\sum_{j=1}^{k} c_{j}\left[\frac{\lambda}{s+\lambda}\right]^{j} .
$$

with $\sum_{j=1}^{k} c_{j}=1$. We consider the case of MGE with $c_{1}=c_{5}=0.25, c_{10}=0.5$, and $\lambda=1$. The $P_{n}(t)$, mean and variance are obtained by the LT method discussed in Section 2.2. Table 1 shows the results for several values of $t$. They are checked against the known results obtained without using roots by Chaudhry [5].

The graph in Figure 1 shows that the $E[N(t)]$ calculated from the obtained distribution functions asymptotically converges to the line

$$
M(t)=\frac{1}{M_{1}} t+\frac{M_{2}-2 M_{1}^{2}}{2 M_{1}^{2}}+o(1)
$$

derived in [16], where $M_{1}$ and $M_{2}$ are the first and

Table 1. $P_{n}(t)$ for MGE distribution with $c_{1}=c_{5}=0.25, c_{10}=0.5$, and $\lambda=1$.

\begin{tabular}{cccccccccccc}
\hline$t$ & $P_{0}(t)$ & $P_{1}(t)$ & $P_{2}(t)$ & $P_{3}(t)$ & $P_{4}(t)$ & $\ldots$ & mean & Chaudhry mean & var & Chaudhry var \\
\hline 1.0 & 0.8411 & 0.1424 & 0.0153 & 0.0012 & 0.0001 & $\ldots$ & 0.1769 & 0.1769 & 0.1843 & 0.1843 \\
2.0 & 0.7706 & 0.1902 & 0.0339 & 0.0047 & 0.0005 & $\ldots$ & 0.2744 & 0.2744 & 0.3025 & 0.3025 \\
3.0 & 0.7157 & 0.2236 & 0.0501 & 0.0090 & 0.0014 & $\ldots$ & 0.3574 & 0.3574 & 0.4047 & 0.4047 \\
5.0 & 0.5959 & 0.2907 & 0.0875 & 0.0208 & 0.0042 & $\ldots$ & 0.5498 & 0.5948 & 0.6183 & 0.6183 \\
10.0 & 0.2363 & 0.4248 & 0.2235 & 0.0822 & 0.0248 & $\ldots$ & 1.2622 & 1.2622 & 1.1013 & 1.1013 \\
50.0 & 0.0000 & 0.0000 & 0.0001 & 0.0056 & 0.0418 & $\ldots$ & 7.4379 & 7.4379 & 4.0784 & 4.0784 \\
\hline
\end{tabular}




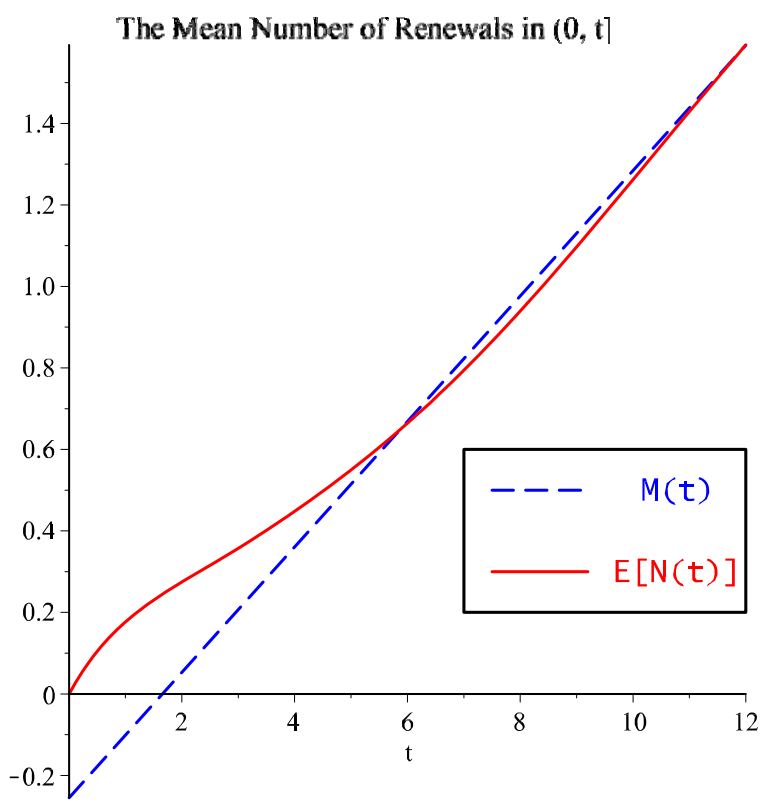

Figure 1. $E[N(t)]$ asymptotically converges to the line $M(t)$.

second moments of $f(t)$. As expected, one can see from this graph that the results given in Equation (6) are good only for large $t$.

\subsection{Matrix Exponential Distribution}

Consider the matrix exponential and non-phase-type dis- tribution (see Bladt [17]) given below. The pdf and its LT are, respectively,

$$
f(t)=\left(1+\frac{1}{4 \pi^{2}}\right)(1-\cos (2 \pi t)) \mathrm{e}^{-t}
$$

and

$$
\tilde{f}(s)=\frac{4 \pi^{2}+1}{(s+1)\left((s+1)^{2}+4 \pi^{2}\right)}
$$

Using partial fractions for transform inversion, we obtain $P_{n}(t)$ for some selected $t$ values. The results are listed in Table 2.

\subsection{Gamma Distribution}

The pdf and LST of the Gamma distribution are given, respectively, by

$$
f(t)=\frac{t^{\alpha-1} \mathrm{e}^{\frac{-t}{\beta}}}{\beta^{\alpha} \Gamma(\alpha)}
$$

and

$$
\tilde{f}(s)=\frac{1}{(1+\beta s)^{\alpha}}
$$

where $\alpha$ and $\beta$ are the shape and scale parameters, respectively. In this example, we set $\beta=1$ and $\alpha=0.55$. The Padé [4/5] approximation of $\tilde{f}(s)$ is given by

$$
\hat{f}(s)=\frac{1+1.97778 s+1.27938 s^{2}+0.29852 s^{3}+0.01804 s^{4}}{1+2.52778 s+2.24340 s^{2}+0.81724 s^{3}+0.10556 s^{4}+0.00232 s^{5}}
$$

The $P_{n}(t)$ are obtained and some selected pmf's, means, and variances are tabulated together with those from Baxter et al. [4] in Table 3.

\subsection{Weibull Distribution}

The pdf

$$
f(t)=\frac{\alpha\left(\frac{t}{\beta}\right)^{\alpha-1} \mathrm{e}^{-\left(\frac{t}{\beta}\right)^{\alpha}}}{\beta}, t>0
$$

and the $\mathrm{CDF}$

$$
F(t)=1-\mathrm{e}^{-\left(\frac{t}{\beta}\right)^{\alpha}}, t>0
$$

of the Weibull distribution do not have the closed-form LT. However, the moments of the Weibull distribution can be obtained from its CDF to use in the Padé method. For $\alpha=3$ and $\beta=1$, we equate moments up to the 8-th moment in the Padé [2/6] approximation, and get

$$
\hat{f}(s)=\frac{1-0.0834 s+0.01200 s^{2}}{1+0.88464 s+0.35058 s^{2}+0.08043 s^{3}+0.01141 s^{4}+0.00097 s^{5}+0.00004 s^{6}}
$$


Table 2. $P_{n}(t)$ for the non-phase-type distribution discussed in Section 3.3.

\begin{tabular}{ccccccccc}
\hline$t$ & $P_{0}(t)$ & $P_{1}(t)$ & $P_{2}(t)$ & $P_{3}(t)$ & $P_{4}(t)$ & $\ldots$ & mean & var \\
\hline 1.0 & 0.3679 & 0.3915 & 0.2154 & 0.0242 & 0.0009 & $\ldots$ & 0.8988 & 0.6785 \\
2.0 & 0.1353 & 0.2881 & 0.3035 & 0.1693 & 0.0832 & $\ldots$ & 1.8411 & 1.5077 \\
3.0 & 0.0498 & 0.1590 & 0.2475 & 0.2322 & 0.1779 & $\ldots$ & 2.7919 & 2.3400 \\
5.0 & 0.0067 & 0.0359 & 0.0919 & 0.1523 & 0.1963 & $\ldots$ & 4.6973 & 3.9929 \\
10.0 & 0.0001 & 0.0005 & 0.0025 & 0.0084 & 0.0215 & $\ldots$ & 9.4619 & 8.1164 \\
\hline
\end{tabular}

Table 3. $P_{n}(t)$ for the Gamma distribution with $\alpha=0.55$ and $\beta=1$.

\begin{tabular}{ccccccccccc}
\hline$t$ & $P_{0}(t)$ & $P_{1}(t)$ & $P_{2}(t)$ & $P_{3}(t)$ & $P_{4}(t)$ & $\ldots$ & mean & Baxter mean & var & Baxter var \\
\hline 0.10 & 0.6871 & 0.2385 & 0.0602 & 0.0119 & 0.0019 & $\ldots$ & 0.4040 & 0.3933 & 0.4623 & 0.4485 \\
0.40 & 0.4071 & 0.3088 & 0.1677 & 0.0743 & 0.0284 & $\ldots$ & 1.0545 & 1.0550 & 1.3970 & 1.3901 \\
1.25 & 0.1291 & 0.1951 & 0.2050 & 0.1730 & 0.1249 & $\ldots$ & 2.6663 & 2.6653 & 4.0487 & 4.0441 \\
\hline
\end{tabular}

Some selected results are listed in Table 4 together with Baxter's (see Baxter et al. [4]). It is noted that when $t$ becomes large, our resulting means and variances match those of Chaudhry [5].

\subsection{Truncated Normal Distribution}

The pdf of the truncated normal distribution is given by

$$
f(t)=\frac{1}{a \sigma \sqrt{2 \pi}} \mathrm{e}^{-\frac{(t-\mu)^{2}}{2 \sigma^{2}}}, t>0
$$

where $a=1-\Phi\left(\frac{-\mu}{\sigma}\right)$, with $\Phi(*)$ being the standard normal distribution function. By the Padé [5/6] approximation

$$
\hat{f}(s)=\frac{1-0.77069 s+0.31300 s^{2}+0.07039 s^{3}+0.00886 s^{4}+0.00048 s^{5}}{1+1.56875 s+1.06454 s^{2}+0.40145 s^{3}+0.08908 s^{4}+0.01108 s^{5}+0.00061 s^{6}}
$$

we are able to obtain $P_{n}(t)$. Some results and comparisons are shown in Table 5.

\subsection{Inverse Gaussian Distribution}

The pdf for the inverse Guassian distribution is

$$
\begin{aligned}
& f(t)=\sqrt{\frac{\lambda}{2 \pi t^{3}}} \mathrm{e}^{-\frac{\lambda(t-\mu)^{2}}{2 \mu^{2} t}}, t \geq 0 . \\
& f(t)=\frac{1}{t \sigma \sqrt{2 \pi}} \mathrm{e}^{-\frac{(\log (t)-\mu)^{2}}{2 \sigma^{2}}}, t \geq 0
\end{aligned}
$$$$
\hat{f}(s)=\frac{1-4.95748 s+9.70859 s^{2}+6.32864 s^{3}+1.57069 s^{4}}{1+5.70748 s+12.33295 s^{2}+12.55938 s^{3}+6.10460 s^{4}+1.27288 s^{5}+0.09280 s^{6}+0.00385 s^{7}}
$$

Some results are shown in Table 6 along with the results of Baxter et al. [4].

\subsection{Lognormal Distribution}

The pdf

and $\mathrm{CDF}$
Setting $\mu=0.75$ and $\lambda=0.5625$, its LST is given by

$$
\tilde{f}(s)=\mathrm{e}^{0.75-0.75(1+2 s)^{0.5}}
$$

where $\lambda$ and $\mu$ can also be expressed in terms of the shape and scale parameters $\phi=\frac{\lambda}{\mu}$ and $\gamma=\frac{\mu^{2}}{\lambda}$, respectively, (see Baxter et al. [4]). By the Padé [4/7] approximation, we get

$$
F(t)=\Phi\left(\frac{\ln t-\mu}{\sigma}\right), \quad t>0
$$

of the lognormal distribution have no closed-form LT. Using the Padé approximation method directly on the lognormal distribution does not lead to the desired results. Our solution is a two-step approximation. In the first step, we sample uniformly $N$ points of $F(t)$, and then connect the adjacent points to form $N$ line segments as the first approximation of the lognormal distribution. The 
Table 4. $P_{n}(t)$ for the Weibull distribution with $\alpha=3$ and $\beta=1$.

\begin{tabular}{cccccccccccc}
\hline$t$ & $P_{0}(t)$ & $P_{1}(t)$ & $P_{2}(t)$ & $P_{3}(t)$ & $P_{4}(t)$ & $\ldots$ & mean & Baxter mean & var & Baxter var \\
\hline 0.25 & 0.9841 & 0.0159 & 0.0000 & & & $\ldots$ & 0.0159 & 0.0156 & 0.0156 & 0.0154 \\
0.60 & 0.8070 & 0.1908 & 0.0022 & 0.0000 & & $\ldots$ & 0.1953 & 0.1965 & 0.1616 & 0.1624 \\
1.00 & 0.3664 & 0.5939 & 0.0391 & 0.0005 & 0.0000 & $\ldots$ & 0.6738 & 0.6724 & 0.3012 & 0.3018 \\
\hline
\end{tabular}

Table 5. $P_{n}(t)$ for the Truncated Normal distribution with $\mu=0$ and $\sigma=1$.

\begin{tabular}{cccccccccccc}
\hline$t$ & $P_{0}(t)$ & $P_{1}(t)$ & $P_{2}(t)$ & $P_{3}(t)$ & $P_{4}(t)$ & $\ldots$ & mean & Baxter mean & var & Baxter var \\
\hline 0.15 & 0.8807 & 0.1121 & 0.0068 & 0.0003 & 0.0000 & $\ldots$ & 0.1267 & 0.1267 & 0.1261 & 0.1261 \\
0.45 & 0.6527 & 0.2849 & 0.0548 & 0.0068 & 0.0006 & $\ldots$ & 0.4178 & 0.4179 & 0.4023 & 0.4024 \\
1.25 & 0.2113 & 0.4002 & 0.2567 & 0.0981 & 0.0268 & $\ldots$ & 1.3507 & 1.3508 & 1.0997 & 1.1007 \\
\hline
\end{tabular}

Table 6. $P_{n}(t)$ for the Inverse Gaussian distribution with $\lambda=0.5625$ and $\mu=0.75$.

\begin{tabular}{ccccccccccc}
\hline$t$ & $P_{0}(t)$ & $P_{1}(t)$ & $P_{2}(t)$ & $P_{3}(t)$ & $P_{4}(t)$ & $\ldots$ & mean & Baxter mean & var & Baxter var \\
\hline 0.25 & 0.7445 & 0.2442 & 0.0112 & 0.0001 & 0.0000 & $\ldots$ & 0.2669 & 0.2715 & 0.2188 & 0.2200 \\
0.70 & 0.3390 & 0.4042 & 0.2062 & 0.0457 & 0.0046 & $\ldots$ & 0.9736 & 0.9739 & 0.7732 & 0.7718 \\
1.25 & 0.1623 & 0.2869 & 0.2867 & 0.1762 & 0.0683 & $\ldots$ & 1.7635 & 1.7638 & 1.5294 & 1.5293 \\
\hline
\end{tabular}

Table 7. $P_{n}(t)$ for the Lognormal distribution with $\mu=0$ and $\sigma=1$.

\begin{tabular}{ccccccccccc}
\hline$t$ & $P_{0}(t)$ & $P_{1}(t)$ & $P_{2}(t)$ & $P_{3}(t)$ & $P_{4}(t)$ & $\ldots$ & mean & Baxter mean & var & Baxter var \\
\hline 0.10 & 0.9855 & 0.0145 & 0.0000 & & & $\ldots$ & 0.0145 & 0.0107 & 0.0143 & 0.0106 \\
0.40 & 0.8226 & 0.1698 & 0.0074 & 0.0001 & 0.0000 & $\ldots$ & 0.1851 & 0.1867 & 0.1664 & 0.1658 \\
1.25 & 0.4113 & 0.4052 & 0.1532 & 0.0274 & 0.0027 & $\ldots$ & 0.8056 & 0.8043 & 0.6636 & 0.6616 \\
\hline
\end{tabular}

uniform sampling step size used is $h=\frac{t_{N}}{N}$, where $t_{N}$ is chosen arbitrarily such that $F\left(t_{N}\right)$ is close to 1 . In addition, the last line segment is modified to reach 1 at $t_{N}$. The resulting first approximation function can be written as

$$
\sum_{i=1}^{N}\left[\frac{F\left(t_{i}\right)-F\left(t_{i-1}\right)}{h}\left(t-t_{i-1}\right)+F\left(t_{i-1}\right)\right]\left[u\left(t-t_{i-1}\right)-u\left(t-t_{i}\right)\right],
$$

where $u\left(t-t_{i}\right), i=1,2, \cdots, N$ are unit step functions. In this example, $\mu$ is set to 0 and $\sigma^{2}$ to 1 . Accordingly, we set $N=100$ and $t_{N}=10$.

In the second step, the LST of the first approximation function is obtained and expanded as a Taylor series. Using the Padé [7/8] method, we have the second approximation of $\tilde{f}(s)$ to be

$$
\hat{f}(s)=\frac{1+2.7966 s+4.7293 s^{2}+4.0493 s^{3}+2.8807 s^{4}+0.9596 s^{5}+0.3004 s^{6}+0.0036 s^{7}}{1+4.3795 s+8.9689 s^{2}+11.2718 s^{3}+9.5600 s^{4}+5.6388 s^{5}+2.2756 s^{6}+0.5795 s^{7}+0.0721 s^{8}}
$$

Table 7 lists selected $P_{n}(t)$, means and variances along with the results of Baxter et al. [4].

\section{Conclusions}

Using Laplace transforms, it is shown that computing the distribution of the number of renewals is straightforward when the LT of the inter-renewal time distribution is rational. For inter-renewal time distributions having nonrational LTs, the Padé method provides good approxima- tions. For the case where the inter-renewal time distributions do not have Laplace transforms, we provide the line segment approximation for the distribution function and apply the Padé method thereafter.

The proposed numerical method is not limited to compute the distribution of number of renewals. It can be applied to other similar processes such as alternating renewal process, the superposition of renewal processes, and cumulative processes, for which Laplace transforms 
are used to solve such problems. Further, the method discussed here can be applied to more complex models such as bulk-renewal processes both in discrete- and continuous-times once the analytic results are known.

\section{Acknowledgements}

The first author acknowledges with thanks for the partial support he received through NSERC \#17287 for carrying out this research.

\section{REFERENCES}

[1] D. R. Cox, "Renewal Theory," John Wiley \& Sons Inc., New York, 1962.

[2] W. Feller, "Probability Theory and Its Applications Vol. II,” Wiley, New York, 1966.

[3] W. Feller, "An Introduction to Probability Theory and Its Applications," Wiley, New York, 1967.

[4] L. A. Baxter, E. M. Scheuer, D. J. McConalogue and W. R. Blischke, "On the Tabulation of the Renewal Functions," Technometrics, Vol. 24, No. 2, 1982, pp. 151-156. doi:10.1080/00401706.1982.10487739

[5] M. L. Chaudhry, "On Computations of the Mean and Variance of the Number of Renewals: A United Approach," The Journal of the Operations Research Society, Vol. 46, 1995, pp. 1352-1364.

[6] J. Abate, G. L. Choudhury and W. Whitt, "A Unified Framework for Numerically Inverting Laplace Transforms," Informs Journal on Computing, Vol. 18, No. 4, 2006, pp. 408-421. doi:10.1287/ijoc.1050.0137

[7] J. Abate, G. L. Choudhury and W. Whitt, "An Introduction to Numerical Transform Inversion and Its Application to Probability Models," In: W. Grassman, Ed., Com- putational Probability, Kluwer, Boston, 1999, pp. $257-$ 323.

[8] J. Abate and W. Whitt, "Numerical Inversion of Laplace Transforms of Probability Distributions," ORSA Journal on Computing, Vol. 7, No. 1, 1995, pp. 36-43. doi:10.1287/ijoc.7.1.36

[9] J. Abate and W. Whitt, "The Fourier-Series Method for Inverting Transforms of Probability Distributions," Queueing Systems, Vol. 10, No. 1-2, 1992, pp. 5-88. doi:10.1007/BF01158520

[10] D. R. Cox and W. L. Smith, "Queues," Methuen and CO Ltd., London, 1967.

[11] L. Kleinrock, "Queueing Systems Volume I: Theory," John Wilery \& Sons, New York, 1975, pp. 212-312.

[12] M. L. Chaudhry and J. G. C. Templeton, "A First Course on Bulk Queues," John Wiley \& Sons Inc., New York, 1983.

[13] G. A. Baker and P. Graves-Morris Jr., "Padé Approximants (Encyclopedia of Mathematics and Its Applications)," 2nd Edition, Cambridge University Press, Cambridge, 1996.

[14] C. M. Harris and W. G. Marchal, "Distribution Estimation Using Laplace Transform," INFORMS Journal on Computing, Vol. 10, No. 4, 1998, pp. 448-458. doi:10.1287/ijoc.10.4.448

[15] E. Parzen, "Stochastic Processes," Holden-Day Inc., San Francisco, 1962.

[16] M. L. Chaudhry and B. Fisher, "Simple and Elegantderivations for Some Asymptotic Results in the DiscreteTime Renewal Process," Statistics and Probability Letters, Vol. 83, No. 1, 2012, pp. 408-421.

[17] M. Bladt, "Computational Method in Applied Probability,” Aalborg University, 1993. 